\title{
Recast-Free Helical Drilling of Fused Silica Using SHG Picosecond Laser Pulses
}

\author{
Chao $\mathrm{He}^{1^{*}}$, Malte Weber ${ }^{2}$, Arnold Gillner ${ }^{1,2}$ \\ ${ }^{1}$ RWTH Aachen University, Chair for Laser Technology LLT, Steinbachstrasse 15, 52074 Aachen, \\ Germany \\ ${ }^{2}$ Fraunhofer Institute for Laser Technology ILT, Steinbachstrasse 15, 52074 Aachen, Germany \\ *Corresponding author's e-mail: chao.he@llt.rwth-aachen.de
}

\begin{abstract}
Fused silica has an excellent optical and thermal properties and therefore is widely used in a variety of industrial applications. With the development of ultrafast laser technology, helical drilling with ultrashort laser pulses has demonstrated great advantages in terms of precision and reproducibility. In this paper, we used a rotating-Dove-prism-based helical optics together with a picosecond laser working at second harmonic generation (SHG). Three types of helical processes: classic helical drilling, helical contour cutting and helical trepanning based on identical helical optics are developed and applied to drill a $200 \mu \mathrm{m}$ hole in $0.5 \mathrm{~mm}$ thick fused silica. The classic helical drilling process has a constant revolution diameter and requires higher pulse energy and longer duration to achieve multiphoton absorption. Therefore, a bulk of recast layer with height of $10 \mu \mathrm{m}$ is generated surrounding the inlet of the micro hole. The height and radial width of recast layer predominantly depend on the focus position and applied pressure of process gas. The helical contour cutting of circular hole can be understood as a combination of helical drilling and circular contour cutting with Computer Numerical Control (CNC) program and precision motion stages. As a result of dynamic cutting front, the molten material are constrained in the cutting kerf and driven out from the hole outlet under pressure of process gas, which contributes to a recast-free drilling. The helical trepanning process is characterized by a through start-hole at beginning and the continuously increased helical diameter of laser pulses during the process. The molten volume within the hole diameter are completely removed and driven out from the pilot-hole. By means of that the drilling process can be accelerated with an enhanced surface quality. Moreover, the entrance of boreholes are free of recast. The influence of the energy deposition in the three drilling strategies on the drilling results regarding the recast layer was investigated by numerical simulation of superposed laser intensity distribution on the helical path.
\end{abstract}

DOI: $10.2961 /$ jlmn.2020.02.2014

Keywords: helical drilling, ultrashort laser pulses, second harmonic generation, fused silica, recast layer, energy deposition

\section{Introduction}

With excellent physical and chemical properties, fused silica has a wide spectrum of scientific and industrial applications such as in optical devices, medical instruments, laser sources and aerospace[1-5]. These application areas are constantly expanding [6]. However, fused silica is a typical hard brittle material and difficult to machine with conventional mechanical methods due to high brittleness, low fracture toughness, and high strength [7]. machining defects such as micro crack and recast layer around the processing site tend to be introduced by conventional techniques as well as ion beam and nanosecond laser processing [8]. The requirements on micromachining of fused silica for optical application is very high. Compared to the conventional glass machining techniques, laser machining offers a number of advantages such as non-contact, high flexibility, high efficiency. Especially, ultrashort pulsed (USP) laser irradiation in the regime of picosecond and femtosecond offers a promising tool to fabricate high precision microstructures in such brittle material.

Over years, processing with ultrashort laser pulses has been considered to be outstanding qualitative associated with low productive due to a low single pulse energy and thus low ablation rate. Modern ultrafast laser sources provide solution to satisfy both sides and they can deliver an average power of up to $1 \mathrm{~kW}$ with pulse duration of several picoseconds and excellent beam quality [9]. In addition, the modern ultrashort pulse laser sources feature harmonic generation unit which converts the fundamental infrared (IR) wavelength into second or higher harmonic. In comparison to the IR radiation, the second harmonic generation ( $\mathrm{SHG}$ ) irradiation qualifies a better focusability and this contributes higher laser energy density in the interaction zone and more effective laser energy deposition. Thus, the employing of SHG laser irradiation in the drilling of micro-scaled boreholes can benefit from higher quality and productivity [10].

With the development of ultrafast laser technology, helical drilling with ultrashort laser pulses has demonstrated great advantages in terms of precision and reproducibility. Thanks to the helical movement of the laser pulses during the drilling process, microholes with high precision can be generate through the layer-by-layer ablation strategy. The helical drilling optics, which is based on a self-rotating Dove prism as beam rotator, has been introduced in our previous 
works [11-14]. During the helical drilling process the ablation front of laser spot moves downwards into the material in a helical path and the material volume ablated with each pulse is much smaller compared to laser trepanning. Thus, this process fulfils an increased accuracy of hole geometry by decreasing temporal and localized thermal load in the workpiece.

In this paper we report the experiment results on fabrication of micro holes in fused silica by using this helical drilling optics with $515 \mathrm{~nm}$ wavelength SHG laser irradiation. The helical drilling optics is able to perform three kinds of drilling processes namely classic helical drilling, helical contour cutting and helical trepanning. The distinguished behavior of laser energy deposition among the helical drilling techniques results to significant distinction in terms of dynamics of melting flows and forming of borehole and thus the morphology of boreholes. To achieve a high precision the energy deposition in helical trepanning and cutting are spatially and temporally adapted. The adaption of energy deposition during the drilling process plays an important role in avoiding the accumulation of thermal effect and reducing recast layer material around the hole inlets. Moreover, the fabrication efficiency can be enhanced.

\section{Experimental setup and materials}

\subsection{UPS laser helical drilling system}

The experimental setup for helical drilling process is schematically shown in Fig. 1. The drilling system consists of three parts: ultrashort pulsed laser source, helical drilling optics and CNC linear motor stages. As laser source a Yb:YAG thin-disk USP Laser (Trumicro 5270, TRUMPF) is employed which has pulse duration of 7 ps and delivers a second harmonic generation of $515 \mathrm{~nm}$ wavelength with a maximum average output power of $60 \mathrm{~W}$ at $400 \mathrm{kHz}$ pulse repetition rate. In helical drilling optics, the helical radius is determined by declination angle of wedge prism which can be precisely controlled by a step motor. Passing through the spinning Dove prism, the laser beam rotates along an optical axis with doubled speed of the hollow shaft motor and is focused by an objective of focal length $60 \mathrm{~mm}$ onto the surface of sample to a spot diameter of approximately $15 \mu \mathrm{m}$. The process material used in this work is UV grade fused silica $\left(\mathrm{SiO}_{2}\right)$ with $0.5 \mathrm{~mm}$ thickness.

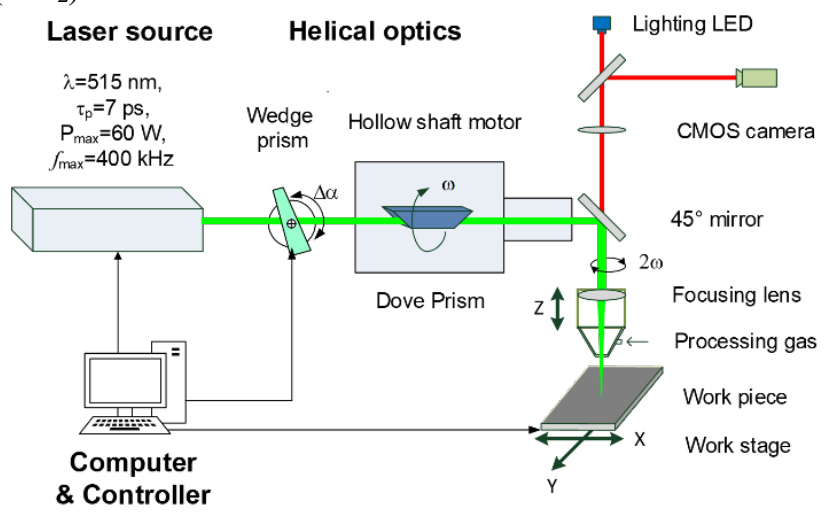

Fig. 1 Schematic diagram of experimental setup for helical drilling processes

\subsection{Process description and parameters}

Three kinds of helical drilling processes: the classic helical drilling, the helical contour cutting and the helical trepanning were can be performed with the helical drilling system. The helical paths of laser spots in three kinds of helical processes are schematically illustrated in Fig. 2. The classic helical drilling has a fixed helical diameter and helical path as shown in Fig. 2(a). The helical contour cutting can be understood as a combination of the classic helical drilling and contour cutting by moving the rotating beam in a designated contour (e. g. a circle with a radius for a circular hole Rc) with assistance of a CNC program. The typical wobbling helical path is shown in Fig. 2(b). The helical trepanning process can be divided in three steps. In the first step, a pilot hole is fabricated in the center by means of a classic helical drilling with a helical radius within the rage of $0-50 \mu \mathrm{m}$, the path is illustrated in a blue circle in Fig. 2(c). Following that, a dynamic drilling process is performed in the second step by enlarging the helical radius continuously until the final geometry is reached. Generally, the dynamic helical drilling process can be defined as a process of helical movements with a dynamically varied helical diameter or center of helical movements. The helical trepanning has a dynamically varied helical diameter and the helical contour cutting process works with fixed helical diameter, but dynamic helical center during the drilling process. Both helical trepanning and helical contour cutting are included in dynamic helical drilling process. The helical path is characterized by a spiral developing contour with a high trace overlap rate. At the end of step 2, the helical radius reaches to the desired dimension. For the last step, a second classic helical drilling process with a larger helical radius is applied for a cylindrical holeprofile and the final contour (red circle in Fig. 2(c)).

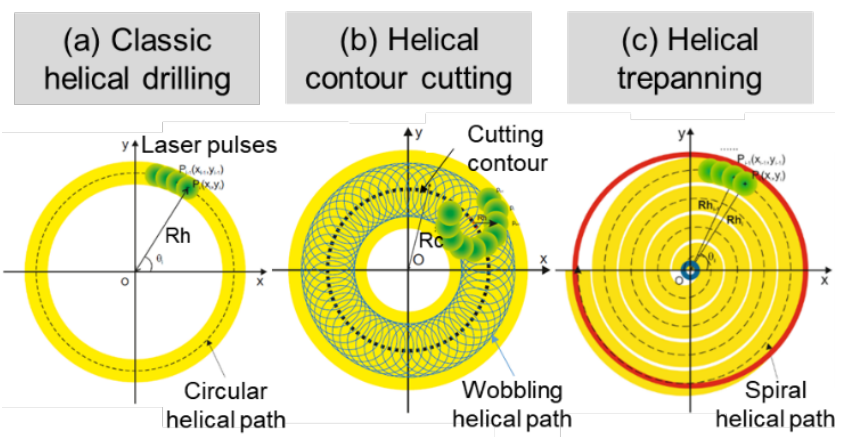

Fig. 2 Paths of laser pulses in three helical drilling processes: (a) helical drilling with a fixed circular helical radius, (b) a superposed path of wobbling helical path and a circular cutting contour in helical cutting, (c) a dynamic spiral helical path in helical trepanning

In order to keep consistence in the comparative study identical single laser pulse energy and frequency as well as process parameter like beam rotation speed, processing gas pressure and focal position were applied in all drilling processes. The helical radius in the classic helical drilling process was fixed at $90 \mu \mathrm{m}$. For helical contour cutting, the helical drilling with helical radius $\mathrm{Rh}$ of $25 \mu \mathrm{m}$ and contour cutting with $75 \mu \mathrm{m}$ cutting radius Rc were applied. In helical trepanning process, the helical radius increases dynamically from $25 \mu \mathrm{m}$ to $90 \mu \mathrm{m}$ with an average speed of $10 \mu \mathrm{m} / \mathrm{s}$. The processing parameters for drilling experiments by means of the three kinds of helical processes are summarized and listed in Table 1. 
Table 1. Processing parameters in the experiments

\begin{tabular}{|c|c|c|c|}
\hline Process & $\begin{array}{l}\text { Classic helical } \\
\text { drilling }\end{array}$ & $\begin{array}{c}\text { Helical con- } \\
\text { tour cutting }\end{array}$ & \multicolumn{1}{|c|}{$\begin{array}{c}\text { Helical } \\
\text { trepanning }\end{array}$} \\
\hline \multirow{2}{*}{$\begin{array}{c}\text { Common } \\
\text { parameter }\end{array}$} & \multicolumn{2}{|c|}{$\begin{array}{l}\text { Laser single pulse energy } 120 \mu \mathrm{J}, \\
\text { Pulse repetition rate: } 40 \mathrm{kHz},\end{array}$} \\
& $\begin{array}{l}\text { Rotation speed of laser beam: } 7200 \mathrm{rpm}, \\
\text { Processing gas pressure: } 3 \mathrm{bar}, \\
\text { Focus position: on front surface of sample }\end{array}$ \\
\hline $\begin{array}{c}\text { Helical } \\
\text { radius }\end{array}$ & $90 \mu \mathrm{m}$ & \multicolumn{1}{|c|}{$25 \mu \mathrm{m}$} & \multicolumn{1}{c|}{ dynamic } \\
\hline $\begin{array}{c}\text { Dynamic } \\
\text { variable }\end{array}$ & none & $\begin{array}{l}\text { Radius of cut- } \\
\text { ting contour } \\
\text { Rc: } 75 \mu \mathrm{m} \\
\text { Feed rate: } 1.5 \\
\mathrm{~mm} / \mathrm{min} .\end{array}$ & $\begin{array}{l}\text { Rotation } \\
\text { speed of } \\
\text { wedge prism: } \\
1.5 \% \text { second }\end{array}$ \\
\hline
\end{tabular}

Geometry and morphology of processed specimens were investigated under an optical microscope and surface topography by confocal laser scanning microscopy (LSM, VK9700, Keyence) and scanning electron microscopy (SEM LEO 1455 EP, Zeiss).

\section{Results and discussion}

\subsection{Superposed laser intensity distribution in helical drilling processes}

The superposed laser intensity (SLI) in the helical drilling processes describes the laser energy deposition of ultrashort pulses on helical path. The superposed laser intensity on the focal plane of laser beam $\mathrm{z}=0$ can be given as [14]:

$$
I_{s}=\sum_{i=1}^{f t} I_{0} \cdot \exp \left\{-2 \frac{d_{h}^{2} \sin ^{2}\left[2 \pi(i-1) \omega_{m} / f\right]}{\omega_{0}^{2}}\right\}
$$

Where, $I_{0}$ is the peak intensity of the laser pulse, $\omega_{m}$ is rotation frequency of Dove prism, $f$ refers to pulse repetition rate, and $d_{h}$ is helical diameter, $\omega_{0}$ is the beam intensity $1 / \mathrm{e}^{2}$ radius of the laser spot on the focal plane. $t$ refers to the drilling time.

Numerical calculation of the superposed intensity distribution of the three kinds of helical processes with the parameters listed in Tab. 1 are carried out and are represented in a color map as shown in Fig. 3.

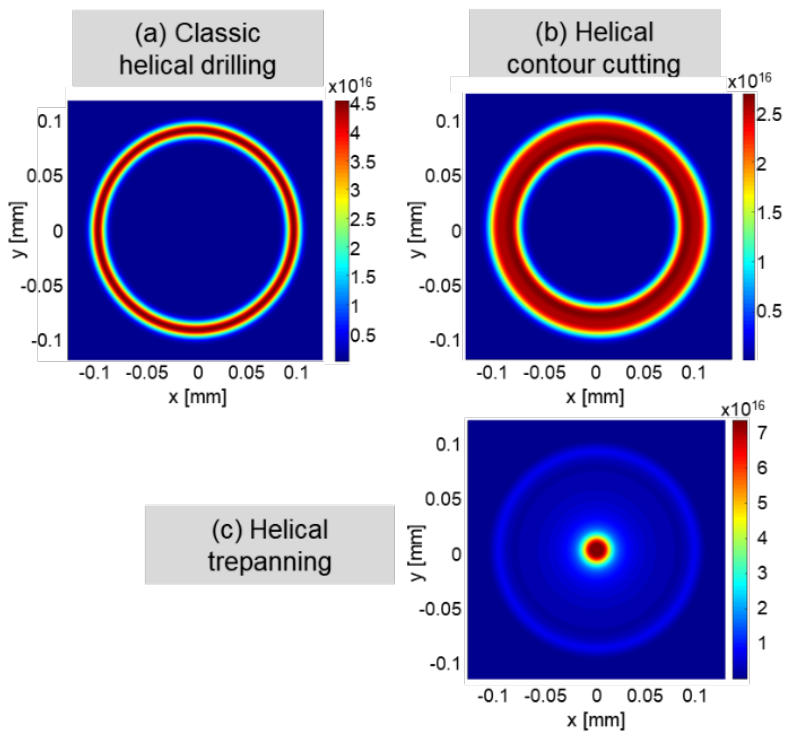

Fig. 3 The superposed laser intensity distribution of the three kinds of helical processes
As a result of the distinguished spatial distribution of the pulse locations, the ablation behavior differs in different helical processes. Fig. 4 depicts the superposed intensity of the three kinds of helical processes in $\mathrm{x} / \mathrm{y}-\mathrm{z}$ plane. The profile of superposed intensity in classic helical drilling can be considered as a symmetric Gaussian distribution and, as a result, a linear accumulation of superposed intensity of laser pulses. In the helical contour cutting process, the appearance of the superposed intensity distribution in case of the circular cutting is analogous to classic helical drilling a donut shape. However, due to a wobbling motion of the laser beam, the intensity at the inner circular contour is higher than that at the outer contour, as can be seen in Fig. 4(b). The helical trepanning is a process with a full-volume ablation. The characteristics of the superposed laser intensity in helical trepanning are, first of all, a peak in the center of the helical path and relative low intensity outside it. Besides a pulse-topulse overlap, a path-to-path overlap in the radial direction at varied helical radii is another key feature of helical trepanning.

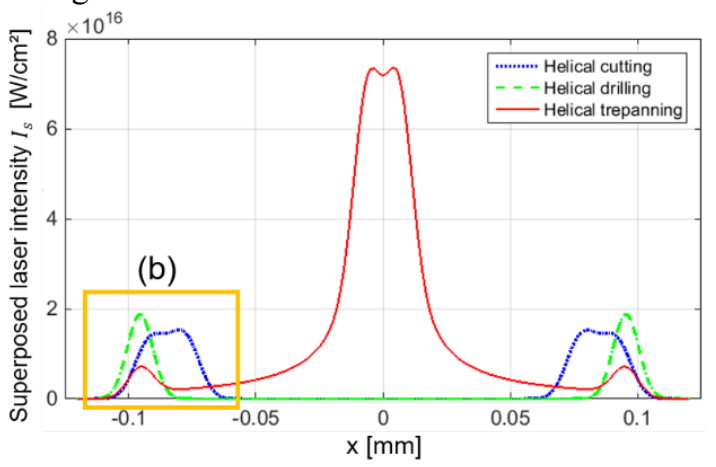

(a)

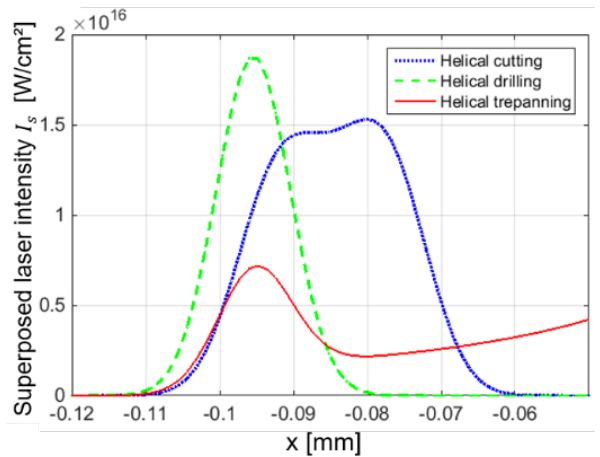

(b)

Fig. 4 Comparison of the superposed laser intensity distribution of classic helical drilling (dashed line in green), helical trepanning (solid line in red) and helical contour cutting (dotted line in blue)

Depending upon the helical radius (the radius of wobbling motion) and feed rate of axis in the helical contour cutting process, the deviation of intensity distribution at inner and outer contours can lead to dissimilar results on, for instance, thermal effect and surface roughness on cutting kerf. A path-to-path overlap caused by the wobbling motion is tangential to the center of cutting contour. Compared to the helical trepanning, the classic helical drilling and the helical contour cutting have a considerably lower ablation volume, The classic helical drilling has the smallest ablation volume comparing to the other two helical processes since merely a single path of laser pulses exists and, therefore, the ablation width is the smallest. 


\subsection{Fabrication of microholes in fused silica by helical processes}

In the classic helical drilling process, the vaporized or molten material by laser radiation must be driven out from the inlet of the borehole before the hole is drilled through. As a result, a part of the mixture of vapors and molten material deposit at the entrance and the recast is formed around the entrance. As soon as a through hole is formed, the fluidic dynamic of the molten material changes. Most of it can be driven out from the exit. The recast at the entrance impairs the quality of the drilling, especially the optical quality of transparent materials. Fig. 5 shows the drilling results in $500 \mu \mathrm{m}$ thick fused silica with three different processes. a number of recast layers at the hole entrance can be clearly seen at the entrance of the hole is fabricated by the classic helical drilling process with a fixed helical diameter of $180 \mu \mathrm{m}$ (Fig. 5(a)). Even after the sample is bathed in ultrasonic cleaner for two minutes, the hierarchy of the recast layers is very clear to be seen. The width of these recast layers reaches nearly $100 \mu \mathrm{m}$ in the radial direction, and the height approximately $8 \mu \mathrm{m}$ as the LSM analysis shows in Fig. 5 . By employing the dynamic helical trepanning process (Fig. 5(b)) little recast layer can be observed on the surface surrounding the hole entrance. The entrance is well-defined and has a sharp edge. Similarly, the helical contour cutting can fabricate the borehole with negligible recast layers as shown in Fig. 5(c).

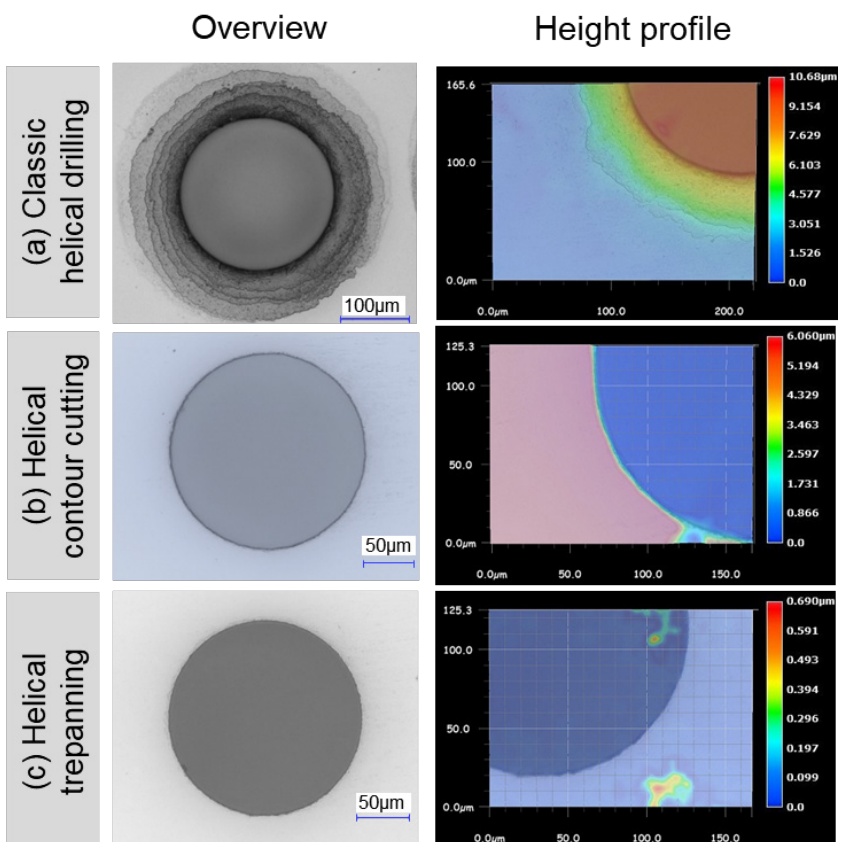

Fig. 5 Comparison of the drilling quality and height of recast layers in fused silica using (a) classic helical drilling, (b) helical contour cutting and (c) helical trepanning process

In analog to hole entrance, the results of the SEM analysis on the exit of the boreholes fabricated by the three kinds of helical processes show the enhanced quality by using the helical trepanning and helical contour cutting as can be seen in Fig. 6. Due to a higher superposed laser intensity on the contour hole entrance in the classic helical drilling, more thermal stress is introduced in the material. As a result, the material around the exit is even chipped by the higher kinetic energy of molten material. No chipping can be seen on the exit of boreholes by dynamic helical processes.

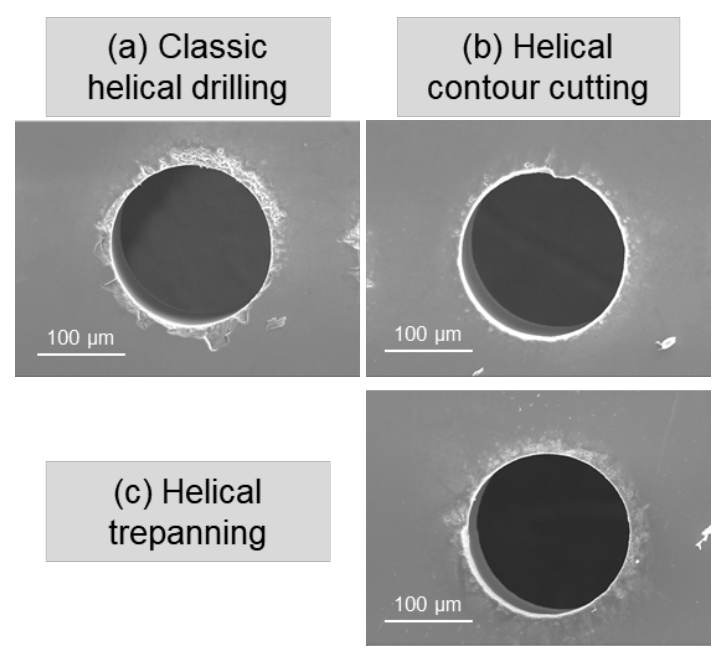

Fig. 6 SEM images of hole exit by (a) classic helical drilling, (b) helical contour cutting and (c) helical trepanning process

\subsection{Analysis on height of recast layer and productivity}

In classic helical drilling process, the molten materials are ejected from the drilling channel and deposit at the entrance. The recast layer is formed as a consequence of the solidification of the molten fused silica. As the ablation front moves deeper in the drilling channel, the range of the spattering of the molten fused silica decreases. Moreover, the recast layers are deposited near the hole entrance and forms a lamination structure, which leads to an increase in height. The height and width of the recast layers at the entrance of the borehole made by classic helical drilling and dynamic helical drilling are characterized by laser scanning microscopy. The comparison of the drilling quality in aspect of height of the recast layer by the classic helical process and dynamic helical process over the hole diameter is plotted and depicted in Fig. 7.

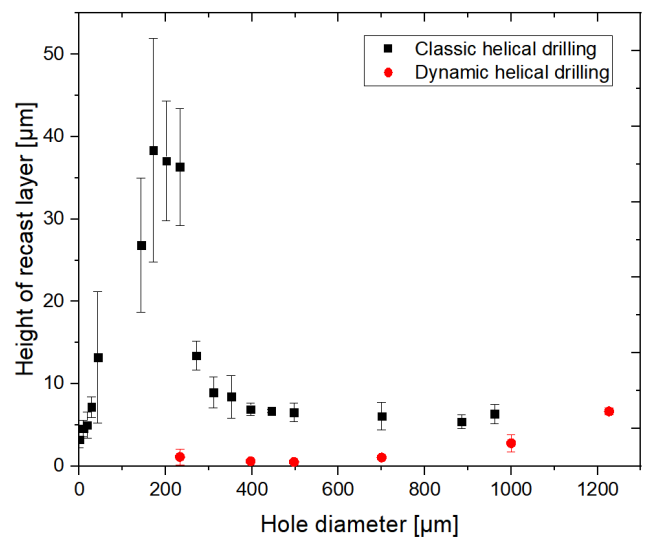

Fig. 7 Comparison of the drilling quality in aspect of height of the recast layer by the classic helical process and dynamic helical process

Apparently, the classic helical drilling process produces much more recast volume than the dynamic helical drilling process. The height of recast layer reaches the maximum in the classic helical drilling of a hole diameter around $200 \mu \mathrm{m}$. The behavior of height of the recast layer with the increase of the hole diameter can be explained by ablation volume by single pulse which is influenced by the laser intensity distribution on the helical path and the thermal accumulation in the ablation volume. In other words, the holes diameter with 
the maximum height of recast layer is determined by the single pulse energy and laser pulse frequency applied as well as the spot diameter of laser beam on the helical path. The ablation volume by laser pulses and the thermal accumulation in the classic helical process vary with the change of the helical diameter. Due to the overlap of the pulses within the area of helical diameter, the ablation volume increases with the hole diameter, whereas the superposed laser intensity in the overlapped area decreases. As soon as the superposed laser intensity in the overlapped area drops below the ablation threshold of the material, an island-like pin appears in the helical drilling process [15]. The ablation volume per pulse reaches the maximum just before the ablation overlap in the middle of the hole appears and the most recast material is generated and thus the height of the recast layer has a maximum at this hole diameter (in the experiment of this work the hole diameter with the maximum height of recast layer is around $200 \mu \mathrm{m}$.

With the further increase of the hole diameter, both the height and the width of the recast layer drop and become stable with an insignificant fluctuation. The recast layer produced in the dynamic helical drilling process can be neglected at drilling with a diameter smaller than $1 \mathrm{~mm}$. Despite that, the recast layer is about $20 \mu \mathrm{m}$ wide along the radial direction of the hole entrance having a diameter of $1 \mathrm{~mm}$. The average height is less than $3 \mu \mathrm{m}$, which is about one third of the recast layer in classic helical drilling.

The dynamic of the molten material by the laser radiation is one of the ways to explain the difference. In the processes of the dynamic helical drilling and helical contour cutting, an initial hole is drilled within one second due to higher concentrated laser energy at a small helical radius. With the relative movement of laser beam to the workpiece, a cutting front is formed with a dynamic direction in the xy plane. Molten material in the volume irradiated by laser pulses will be driven out from the exit of cutting kerf with assistance of the processing gas (3 bar).

Furthermore, the thermal accumulation during the drilling processes is the other reason for the formation of the recast layer. The laser pulse energy absorbed by material can be divided into different parts: the ablation energy for material evaporation and kinetic and thermal energy in the vapor, and the residual heat [16]. With greater density of laser pulses in ablation area, the deposited residual heat does not fully dissipate, accumulates and heats the base material; finally a certain amount of residual heat attributes to ablation. In this case, the efficiency of ablation energy is greater than that in a larger helical diameter and lower overlapping degree. It can also explain why the ablation rate is higher at the smaller helical diameter than at the larger helical diameter. The ablation in the classic helical drilling with a helical diameter of $200 \mu \mathrm{m}$ produces a large number of recasts due to a large amount of local thermal accumulation

The drilling efficiency by the classic helical process, helical trepanning and helical contour cutting of fused silica are quantitatively characterized as shown in Fig. 8.

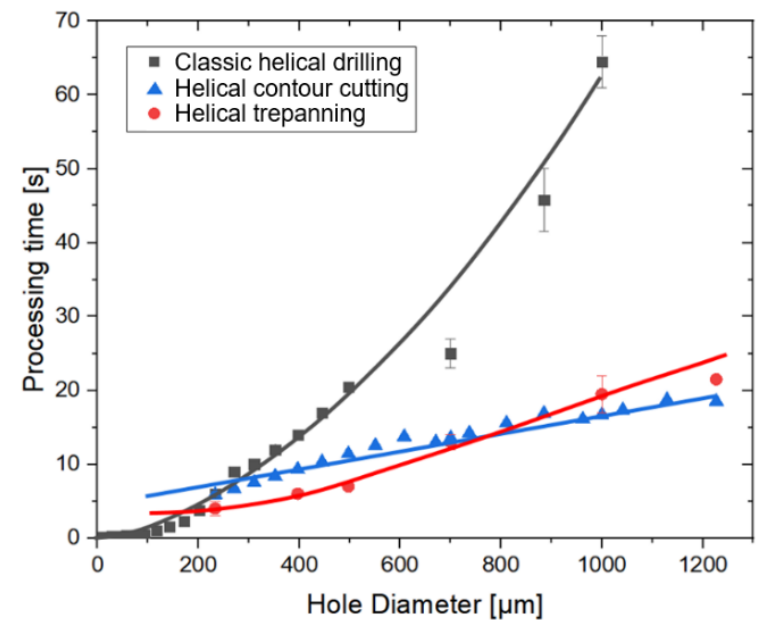

Fig. 8 Comparison of the drilling efficiency by the classic helical process, dynamic helical process and helical contour cutting of fused silica

The adaption of the dynamic of the molten material in the dynamic helical drilling process and the helical contour cutting process not only contributes to reducing of recast layer volume, but also enhances the drilling efficiency. The classic helical drilling as a static process is more efficient in fabricating microholes with diameters smaller than about $170 \mu \mathrm{m}$. However, the increase in hole diameter demands a longer drilling time and more laser pulses by the classic helical drilling than the dynamic helical drilling processes. The fabrication of a $1 \mathrm{~mm}$ diameter hole requires approximately 60 seconds. Due to that the molten material is fast driven out from the pilot hole, the drilling time for the larger hole diameter is significantly reduced to between 15 seconds and 20 seconds by means of dynamic helical drilling and helical contour cutting process.

Fittings of processing time over different hole diameters are done to show the development of processing efficiency by different helical processes. For classic and dynamic helical drilling, a polynomial fitting is applied. For the helical contour cutting a linear fitting is used since the processing time is linear to the perimeter of the hole. The fitted lines divide the hole diameter into three zones and indicate the most efficient drilling process in each zone. For the microhole having a diameter smaller than $170 \mu \mathrm{m}$, the classic helical drilling process should be chosen. For diameter between $170 \mu \mathrm{m}$ and ca. $800 \mu \mathrm{m}$, the processing efficiency as well as the drilling quality can be enhanced by employing dynamic helical drilling. With the increase of hole diameter, the superposed laser intensity on the helical path of final diameter decreases and leads to a slower ablation rate and thus longer processing time. The helical contour cutting has a constant helical diameter and thus the superposed laser intensity is consistent over the whole cutting contour. Therefore, the processing time increases linearly with the hole diameter. In the experiment of this work, the helical contour cutting process has advantage over the other helical processes in fabrication holes with diameter larger than $800 \mu \mathrm{m}$.

\section{Conclusion}

Three kinds of helical drilling processes i.e. the classic helical process, the helical trepanning and the helical contour cutting in fused silica by using Dove-prism-based helical drilling optics and SHG ultrashort pulsed laser are demonstrated. The superposed laser intensity distribution of 
the helical drilling processes has distinguished characteristics and play a determinative role in the energy deposition and further the drilling results.

By taking advantage of the dynamic helical drilling process, the performance of the available helical optics can be improved. First of all, the height and width of recast layer deposited surrounding the hole entrance can be minimized. Moreover, by adapting the dynamic of molten material in the drilling channel, the drilling speed for hole diameter $>$ $170 \mu \mathrm{m}$ can be increased by means of the helical trepanning and helical contour cutting. Meanwhile, the drilling quality with respect to recast layer can be significantly enhanced.

As the final remark, owing to the change of molten dynamic in the borehole, the advantages by using dynamic helical process apply not only to the glass material but to other dielectric material and metallic materials with different thickness.

\section{Acknowledgments}

This work is funded by the Deutsche Forschungsgemeinschaft (DFG, German Research Foundation) under Germany's Excellence Strategy - EXC-2023 Internet of Production -390621612

\section{References}

[1] H. Song, J. Dan, X. Chen, J. Xiao, and J. Xu: Int. J. Adv. Manuf. Technol., 97, (2018) 267.

[2] J. Zhang, K. Sugioka, and K. Midorikawa: Appl. Phys. A, 69, (1999) 879.

[3] R. Taylor, C. Hnatovsky, and E. Simova: Laser Photonics Rev., 2, (2008) 26.
[4] L. Sudrie, A. Couairon, M. Franco, B. Lamouroux, B. Prade, S. Tzortzakis, and A. Mysyrowicz: Phys. Rev. Lett., 89, (2002) 186601.

[5] J. Ihlemann, B. Wolff, and P. Simon: Appl. Phys. A, 54, (1992) 363

[6] R. French and H. Tran: Annu. Rev. Mater. Res., 39, (2009) 93.

[7] C. Zhou, Q. Zhang, C. He, and Y. Li: Optik, 125, (2014) 4064.

[8] S. Nikumb, Q. Chen, C. Li, H. Reshef, H. Zheng, H. Qiu, and D. Low: Thin Solid Films, 477, (2005) 216.

[9] J.-P. Negel, A. Loescher, A. Voss, D. Bauer, D. Sutter, A. Killi, M. A. Ahmed, and T. Graf: Opt. Express, 23, (2015) 21064.

[10] M. Kraus, M. A. Ahmed, A. Michalowski, A. Voss, R. Weber, and T. Graf: Opt. Express, 18, (2010) 22305.

[11] C. He, J. Bühring, and A. Gillner: Procedia CIRP, 74, (2018) 305

[12] H. Sakurai, C. He, K. Konishi, H. Tamaru, J. Yumoto, M. Kuwata-Gonokami, and A. Gillner: J. Appl. Phys., 125, (2019) 173109

[13] F. Zibner, C. Fornaroli, J. Holtkamp, J. Ryll and A. Gillner: Proc. SPIE Int. Soc. Opt. Eng., 9582, (2015) 95820C.

[14] C. He, D. Esch and A. Gillner: J. Laser Micro/Nanoeng., 14, (2019) 173.

[15] C. He, C. Hartmann, C. Fornaroli, F. Zibner, and A. Gillner: Proc. LiM., (2015) 1.

[16] F. Bauer, A. Michalowski, T. Kiedrowski, and S. Nolte: Opt. Express, 23, (2015) 1035.

(Received: July 17, 2020, Accepted: September 15, 2020) 